Setting This study was undertaken at the Malta Football Association (MFA), responsible for the Maltese national football teams.

Participants 13 semi-structured interviews with Maltese female and male national team football players $(n=7)$, coaches $(n=3)$, and health professionals $(n=3)$ were conducted. Data were analysed inductively using thematic analysis.

Results Three themes were identified: (1) How do I perceive an injury? consisted of various constructs of a sports injury, yet commonly defined based on performance limitations. (2) How do I deal with an injury? encapsulated the process of managing the injury (3) What influences my perception, reporting and management of an injury? comprised personal and contextual factors that influenced the perception and, consequently, the management of an injury.

Conclusions The injury surveillance system implemented within the Maltese national football team should make use of performance limitations as the main injury definition. Injury perception, reporting and management are influenced by the socio-ecological context. In acknowledging this influence, ongoing human interaction should be involved between stakeholders in all the processes of the injury surveillance system, emphasising its active role to optimise the players' injury risk mitigation and management.

\section{OVERALL, REGION- AND TISSUE-SPECIFIC INJURY RATES IN UK SUMMER OLYMPIC MALE AND FEMALE ATHLETES}

\footnotetext{
1,2Moses Wootten, 'Steve McCaig, 'Anita Biswas, 'Michael Brownlow, 'Lee Herrington, ${ }^{2}$ Abbie Taylor, ${ }^{2}$ Freddie Brown, ${ }^{1}$ Richard Burden, ${ }^{1}$ Craig Ranson. 'English Institute of Sport, London, UK; ${ }^{2}$ UK Sport, London, UK
}

\subsection{6/bjsports-2021-IOC.210}

Background Existing studies have reported that female athletes are at increased risk of particular injury types e.g. ACL rupture and bone stress injury, and have reduced risk of others e. g. hamstring injury. Other sport-specific studies have reported that female athletes generally have higher risk of injury in some sports, lower risk in others. No previous study has compared male and female injury rates across a range of summer Olympic sports.

Objective To determine whether male and female athletes experience injuries at different rates within the UK High Performance System.

Design Retrospective cohort study.

Setting UK summer Olympic sports.

Participants 914 athletes (439 female, 475 male).

Interventions There was no intervention.

Main Outcome Measurements Incidence rate ratio (IRR) comparing overall injury rates (as incidence per athlete year) and risk ratio (RR) comparing overall injury burden (training days restricted or unavailable per athlete year) for male and female athletes. The IRR and RR were also compared for injuries in different regions as well as different tissue types.

Results Female athletes had both a greater overall injury rate (females 2.6 injuries per athlete year, males 2.1; IRR 1.23, $95 \%$ CI 1.17 to $1.2330, \mathrm{p}<0.001$ ) and overall injury burden (females 42.58 days training affected per athlete year, males 35.0; RR 1.211.22, 95\% CI 1.201.21 to $1.231 .24, \mathrm{p}<0.001$ ) than male athletes. Female athletes have a higher risk of lumbar/pelvis, foot and ankle injuries (among others) and a higher risk of superficial/skin, ligament/joint and bone injuries.
Conclusions Female athletes are at greater risk of suffering an initial injury with more subsequent training time-loss than male athletes. The higher risk of ligament/joint and bone injuries supports previous analyses of gender-specific injury rates. Further work is required to determine causal mechanisms so that tailored mitigation strategies can be developed for female and male athletes.

\section{UNDERSTANDING HEALTH PROBLEMS OF BASKETBALL REFEREES}

Daniela Annanias Gimenes de Paula, Luiz Augusto Borges Gomes, Ronaldo Alves da Cunha, Carlos Vicente Andreoli. Universidade Federal de São Paulo, São Paulo, Brazil

\section{$10.1136 /$ bjsports-2021-IOC.211}

Background Basketball referees are responsible for rule length and decision making. They have high physical and psychological demand during the match.

Objective To understand the health problems of basketball officials, such as disorders or musculoskeletal injuries and their complaints.

Design Prospective cohort study.

Setting Referees of Basketball Federation of São Paulo.

Patients (or Participants) Initially, 78 referees were assessed, over 18 years old, both sexes and with more than 1 year of practice. Participants who refused to participate were not included, and participants who refused to stay in the study during follow-up were excluded. The participants number varied over the follow-up weeks, with at least 42 participants.

Interventions (or Assessment of Risk Factors) An initial assessment of the basketball referees was made at the annual physical assessment day of Basketball Federation of São Paulo and then they were followed online weekly for 12 weeks.

Main Outcome Measurements The initial assessment was made using an assessment form with personal data, practice and injury historic and knowledge about injury prevention. The weekly follow up was done using the questionnaire Oslo Sports Trauma Research Center (OSTRC) questionnaire to assess health problems.

Results Mean age was 36.5 ( \pm 9.8) years, 66 (84.6\%) were male. The most reported injuries by the referees in the 3 months prior the initial assessment were knee injuries (26.9\%), mainly at the time of fitness training (65.4\%). During the follow-up, musculoskeletal injuries had weekly mean prevalence of $17.4 \%(16.5$ - 18.3) versus 3.2\% (0.4 - 6.0) for illness. Regarding injuries, the ankle was the most affected region for acute injuries and the knee for overuse injuries.

Conclusions Basketball referees were mainly affected by lower limb injuries. Considering the injury profile and the prevalence of associated musculoskeletal complaints found in this study, it appears that injury prevention programmes should be developed and integrated into the fitness training routine of the referee.

\section{INJURY PREVALENCE IN DUTCH HANDBALL OVER THE SEASON 2018-2019}

${ }^{1}$ Maarten Barendrecht, ${ }^{1}$ Tim Arnts, ${ }^{2}$ Linda van Maanen-Coppens. 'Avanst, Breda, Netherlands; ${ }^{2}$ Dutch Handball Federation, Arnhem, Netherlands

10.1136/bjsports-2021-IOC.212 
Background Dutch handball has gained popularity since recent international successes. Downside is the risk of injuries.

Objective To gather information on the prevalence and distribution of injuries in Dutch handball for the development and implementation of targeted preventive measures.

Design Repeated cross-sectional design.

Setting Adolescent and adult handball players active in Dutch leagues of any playing level were invited through social media, club mailings and the website of the Dutch Handball Federation to participate in a monthly online survey.

Participants Handball players (16 years or older) were included. In total 1136 respondents (80\% female) filled in 4171 monthly questionnaires from September 2018 to June 2019.

Risk Factors Outcomes were categorized by sex and playing position.

Main Outcome Measurements New injuries (injury incidence) and number of injured players divided by the total number of respondents (prevalence) per body location. Injury definition: 'the inability to fully participate in handball training and/or matches over the last month due to a problem sustained during handball. The Oslo Sports Trauma Research Centre Questionnaire (OSTRCQ) was used to quantify injury severity.

Results Of 657 new injuries, the knee (21\%), ankle (17\%) and shoulder (10\%) contributed similarly in both sexes and the lower leg predominantly in female players $(10 \%)$. Overall injury prevalence was $43 \%$ (female 46\%, male 34\%). Most prevalent body locations and their mean (standard deviation (sd)) OSTRCQ-scores were the knee (8.8\%; OSTRCQ 61, sd 26), shoulder (4.4\%; OSTRCQ 50, sd 22), ankle (4.0\%; OSTRCQ 55, sd 27) and the lower leg (3.1\%; OSTRCQ 56, sd 26). For all playing positions the knee showed the highest prevalence followed by the shoulder (wings and backs) and/or ankle (backs, pivots and goalies).

Conclusions The high prevalence of knee, ankle and shoulder injuries in both sexes in Dutch handball emphasizes the need for implementation of preventive measures. Preventive training programs already proven successful in handball populations from other countries can be implemented in Dutch handball as well.

\section{INJURY PREVENTION POLICIES IN DUTCH HANDBALL CLUBS}

${ }^{1}$ Maarten Barendrecht, 'Davey Driessen, ${ }^{2}$ Linda van Maanen-Coppens. 'Avans+, Breda, Netherlands; ${ }^{2}$ Dutch Handball Federation, Arnhem, Netherlands

\subsection{6/bjsports-2021-IOC.213}

Background To reduce the number of Handball injuries implementation of preventive measures on all organisational levels is necessary. In Dutch handball clubs, many youth teams are trained/coached by volunteers. Qualified trainers at senior level come and go.

Objective To investigate (barriers and facilitators to) current preventive policies in Dutch handball clubs.

Design Cross-sectional survey.

Setting An online survey was sent to all (371) Dutch handball clubs in February 2018.

Participants Technical committee members/head coaches of sixty-seven clubs (18\% response), covering all competition levels, answered the questionnaire. Units of investigation: Handball clubs: management and trainer level

Main Outcome Measurements Club policies regarding injury prevention and barriers and facilitators for implementation on club level.

Results Of all responding clubs, 20\% had a club policy on injury prevention, in $51 \%$ the choice was up to trainers themselves and the other $29 \%$ had no clear knowledge of a policy on injury prevention. Only 14\% of all clubs had a long-term policy on maintenance of preventive measures. On average $30 \%$ of the active trainers in a club had a trainer's licence of any level. Important barriers for implementation were insufficient knowledge on injury prevention in trainers and/or within the club and lack of implementation competencies within the club. Other barriers were financial, organisational and the high number of volunteers. Insight in the contents and effectiveness of preventive measures by means of an app and/or yearly in-company workshops were suggested strategies to facilitate implementation. Furthermore, external support from the Dutch Handball Federation and support for the implementation process in the club as well as incorporating prevention within athlete development were mentioned.

Conclusions Injury prevention policies seem to be scarcely implemented in Dutch handball clubs although interpretation of these results must take the $18 \%$ response rate into consideration. Externally supported knowledge transfer and implementation management on club level are needed to facilitate the implementation of injury prevention in Dutch handball clubs.

\section{DESCRIPTION OF THE CONTEXT FOR INJURY PREVENTION INTERVENTIONS DEVELOPMENT AND IMPLEMENTATION IN YOUTH BRAZILIAN BASKETBALL: A CROSS-SECTIONAL STUDY}

Luiz Augusto Borges Gomes, Carlos Vicente Andreoli, Ronaldo Alves da Cunha, Helena Santos de Oliveira, Benno Ejnisman, Moises Cohen. Sports and Traumatology Center, Department of Orthopedics and Traumatology, Federal University of São Paulo, São Paulo, Brazil, São Paulo, Brazil

\subsection{6/bjsports-2021-IOC.214}

Background The implementation of injury prevention interventions commonly do not consider the context where injury occurs.

Objective To describe the socioecological context to guide the development and implementation of injury prevention interventions in youth Brazilian basketball (YBB).

Design Cross-sectional study.

Setting YBB teams affiliated to the Basketball Federation of the State of São Paulo. Participants recruitment and data collection occurred during the regular season, between March and September 2018.

Patients (or Participants) 534 athletes and 54 staff members of 35 YBB teams were included in the study. Eligibility criteria included (1) staff members who have graduated in a health care profession and (2) youth athletes with age between 10 and 19 years old.

Interventions (or Assessment of Risk Factors) Participants responded a survey on a regular day of practice or official match. 